\title{
Evaluación de las creencias hacia la atención de personas con discapacidad auditiva, en prácticas a distancia de Educación Física de estudiantes universitarios durante la pandemia por COVID-19 \\ Evaluation of beliefs towards the care of people with hearing disabilities, in online practices of Physical Education of university students during the COVID-19 pandemic

\author{
*,**Paulina Yesica Ochoa-Martínez, ${ }^{*, * *}$ Javier Arturo Hall-López, ${ }^{*}$ Edgar Ismael Alarcón-Meza, ${ }^{* *}$ Ana Maria Teixeira \\ *Universidad Autónoma de Baja California (México), **Universidad de Coímbra (Portugal).
}

\begin{abstract}
Resumen. Objetivo: Evaluar las creencias hacia la atención a la discapacidad en actividad física, en estudiantes de Licenciatura en Actividad Física y Deporte al realizar prácticas docentes de Educación Física a distancia durante la pandemia por COVID-19 a escolares con discapacidad auditiva. Método: Mediante un enfoque cuantitativo cuasiexperimental, se capacitó a 17 estudiantes de Licenciatura en Actividad Física y Deporte de la Facultad de Depor tes de la Universidad Autónoma de Baja California, que realizaron prácticas docentes, implementando 12 clases de Educación Física a distancia a escolares con discapacidad auditiva, planeando, diseñando y aplicando estrategias pedagógicas acordes a su condición. Antes y después se evaluaron las actitudes sociales y profesionales hacia las personas con discapacidad. Resultados: El análisis estadístico con test t-Student para muestras independientes reportó diferencias significativas ( $<<0.05)$, el promedio de los 30 ítems del instrumento antes de las prácticas docentes fue 2.6 y después de las prácticas docentes 1.9 con un porcentaje de cambio de -29 Ä \%. Conclusiones: Se mejoraron las creencias hacia la atención a la discapacidad a través de la actividad física, en un ambiente educativo a distancia, que es favorable en el proceso de enseñanza-aprendizaje de los estudiantes participantes, lo cual favorecerá competencias futuras para el desarrollo de intervenciones efectivas y adecuadas en poblaciones con discapacidades.

Palabras clave: Actitud; Discapacidad auditiva; Educación Física, Educación Especial; Educación en línea.
\end{abstract}

\begin{abstract}
Objective: To evaluate the beliefs towards attention to disability in physical activity, in students of Bachelor's Degree in Physical Activity and Sport when carrying out virtual Physical Education teaching practices during the COVID-19 pandemic to schoolchildren with hearing disabilities. Method: Through a quasi-experimental quantitative approach of a quantitative type, 17 undergraduate students in Physical Activity and Sports from the Faculty of Sports of the Autonomous University of Baja California were trained, who carried out teaching practices, implementing 12 classes of Physical Education online to schoolchildren with hearing disability, planning, designing and applying pedagogical strategies according to their condition. Before and after, social and professional attitudes towards people with disabilities were evaluated. Results: The statistical analysis with $\mathrm{t}$-Student test for independent samples reported significant differences $(\mathrm{p}<0.05)$, the average of the 30 items of the instrument before the teaching practices was 2.6 and after the teaching practices 1.9 with a change percentage of -29 $\ddot{\mathrm{A}} \%$. Conclusion: Beliefs towards attention to disability were improved through physical activity online educational environment, which is favorable in the teaching-learning process of participating students, providing elements for the development of future effective and adequate interventions in populations with disability.
\end{abstract}

Keywords: Attitude; Hearing Impairment; Physical Education; Special Education; Online Education.

\section{Introducción}

La discapacidad auditiva forma parte de la clasificación internacional del funcionamiento (CIF) de la discapacidad y la salud de la Organización Mundial de la Salud (OMS), y se establece como un déficit total o parcial de la percepción que se evalúa de la pérdida del audio en cada oído (OMS, 2001). El informe mundial sobre la discapacidad, reporta que el número de personas con esta condición está creciendo y principalmente en sectores sociales vulnerables, además se menciona que el estudiantado con discapacidad tienen menos pro-

Fecha recepción: 18-05-21. Fecha de aceptación: 22-08-21 Javier Arturo Hall-López javierhall@uabc.edu.mx babilidades que sus homólogos sin discapacidad diagnosticada de ingresar en la escuela, permanecer en ella y superar los cursos sucesivos (Varguillas y Bravo, 2018); la Organización de las Naciones Unidas para la Educación, la Ciencia y la Cultura (UNESCO), al proponer que la Educación Física (EF) dentro de sus políticas garantice el centrar metodologías inclusivas, que fomenten y sensibilicen en general los valores de la Educación Física, deben ser elaboradas y utilizadas para normalizar, la participación de alumnos, padres y miembros de la comunidad (UNESCO, 2015); una revisión sistemática correlaciona menores práctica de actividad física en escolares con la presencia de discapacidad (Li, et al., 2016), de igual manera un estudio con diseño meta analítico, reportó que los escolares sin discapacidad realizan con mayor frecuencia, intensidad y duración actividad física 
que los escolares sin discapacidad (Jung, Leung, Schram yYun, 2018).

En la actualidad, debido a la crisis de salud por COVID-19, se reporta que la actividad física en personas con discapacidad física ha disminuido y el distanciamiento social como medida para propagar la infección ha sido una barrera para la práctica deportiva (Blocken, Malizia, vanDruenen y Marchal, 2020; Lebrasseur, et al., 2021). Se estima que como resultado de la pandemia por COVID-19, aumentarán las enfermedades cardiovasculares en la población (Lippi, Henry y SanchisGomar, 2020), y en el estudiantado de edad escolar se incrementará el peso corporal (Rundle, Park, Herbstman, Kinsey y Wang, 2020), en ese sentido se considera al profesorado de EF con las competencias adecuadas para generar estrategias de salud incrementando la actividad física en la población (Webster, et al., 2015), y en la actual pandemia para minimizar la vulnerabilidad a la infección por COVID19 se recomienda realizar de manera regular de actividad física de intensidad moderada a vigorosa (Chen, Mao, Nassis, Harmer, Ainsworth y Li, 2020).

Derivado de la pandemia por COVID-19, en el contexto educativo, se adecuó de manera súbita la EF de entornos presenciales a entornos a distancia (Hall-López y Ochoa-Martínez, 2020), en México, se reporta que la clase de EF durante la crisis de salud por COVID-19, se implementó principalmente con metodología de aula invertida (González, Gastélum-Cuadras, Velducea, González y Domínguez, 2021), en el ámbito universitarios, la formación de recurso humano entorno a la actividad física y deporte, también se reportan adecuaciones para realizar prácticas en EF mediante entornos a distancia sincrónicos y asincrónicos (Hall-López, OchoaMartínez y Alarcón, 2021).

Las instituciones de educación superior, al formar recurso humano, presentan en sus planes de estudio contenidos incluyentes, dentro de los cuales está dar atención especializada a personas con discapacidad (Alcaín y Medina-García, 2017), una investigación que presenta dicho abordaje, evaluó la actitud docente en la enseñanza de la actividad física en personas con discapacidad auditiva (Ochoa-Martínez, 2020a), la cual se toma como referencia en esta investigación tomando en cuenta que la actitud del profesorado hacia la discapacidad, se ha considerado como un elemento clave de éxito en el proceso enseñanza aprendizaje (Camaño, Rodríguez, Rojas, Erazo y Pancho, 2019), se han reportan dificultades en el proceso de enseñanza a distancia, la evaluación de los aprendizajes (Baena-Morales, López-Morales y
García-Taibo, 2020), se relatan implicaciones negativas para adaptarse al proceso enseñanza aprendizaje durante la pandemia por COVID-19 (Hortigüela-Alcalá, Garijo y Pérez-Pueyo, 2021).

En la investigación, se realizaron adecuaciones curriculares para adaptarse a la educación a distancia, tomando en cuenta el plan de estudios de Educación Física establecido por la Secretaría de Educación Pública, (SEP, 2017), como órgano rector de la educación en México se enfatizó, los ámbitos del perfil de egreso denominados habilidades digitales y atención al cuerpo y la salud, fundamentando esta conexión y enlace de necesidad social con los contenidos curriculares de planes de estudios dedicados a la enseñanza de la EF que en esta pandemia se ha realizado a distancia (Hall-López y Ochoa-Martínez, 2020), en esta investigación se toma en como referencia adecuarse a un entorno a distancia aplicándose en escolares con discapacidad auditiva, que requieren estrategias pedagógicas específicas para promover el desarrollo motor y la actividad física, adaptando la comunicación y herramientas didácticas que faciliten el currículo (Fiorini y Manzini, 2018; Barboza, Ramos, Abreu, y Castro, 2019), por lo anterior el objetivo del presente estudio es evaluar las creencias hacia la atención a la discapacidad en actividad física en prácticas docentes durante la pandemia por COVID-19 en futuros licenciados en actividad física y deporte.

\section{Método}

\section{Diseño}

El estudio fue registrado por el Cuerpo Académico Ciencias de la Actividad Física y Deporte clave CACUABC-175 de la Facultad de Deportes, Campus Mexicali y se aprobó con el código 149/2606, ante la Coordinación General de Posgrado e Investigación de la Universidad Autónoma de Baja California, localizada en México, mediante un enfoque cuantitativo cuasiexperimental, siguiendo los principios éticos de investigación en seres humanos de la declaración de Helsinki.

\section{Participantes}

Los sujetos participantes fueron, 17 estudiantes de Licenciatura en Actividad Física y Deporte (10 hombres y 7 mujeres con una edad promedio de $23 \pm 4.5$ años), que cursaban la unidad de aprendizaje de séptimo semestre, denominada actividad física para necesidades educativas especiales que recibieron capacitación teórica, para posteriormente realizar prácticas en un centro de atención múltiple que da atención educativa a esco- 
lares con discapacidad auditiva diagnosticada.

\section{Instrumentos}

Las creencias hacia la atención a la discapacidad en actividad física, de los estudiantes de Licenciatura en Actividad Física y Deporte, se evaluaron antes y después de la capacitación y práctica docente, mediante la escala de creencias hacia la atención a la discapacidad en actividad física (González y Baños, 2012).

El instrumento evalúa elementos actitudinales, está constituida por 30 reactivos a manera de escala tipo Likert divididos en cuatro sub escalas: Creencias de control percibido: ítems 1, 13, 17, 18 y 24 («Me sentiría incómodo trabajando con compañeros con discapacidad»); Creencias sobre la actitud: 2,3,8, 19,20,21,22,23,26,27,29 y 30 («Sobre discapacidad preferiría que me asesorara un especialista a un compañero); Creencias generalizadas: 4,5,6,7,9,10,11 y 12 («los alumnos con discapacidad se sienten más cómodos en las aulas de Educación Especial»); y Creencias intencionales: 14,15,16 y 25 («Preferiría no tener alumnos con discapacidad en mi clase») . La puntuación cercana a 1 para cualquier ítem de la escala refleja una actitud positiva ante las personas con discapacidad. Estableciendo los valores de 1 como no de acuerdo a 5 muy de acuerdo. La escala fue diseñada para investigación ad hoc y la fiabilidad obtenida fue de un alpha de Cronbach de 0.78 (González y Baños, 2012).

\section{Procedimientos}

Se realizó capacitación teórica, previa a impartir las clases de EF a distancia a los escolares con discapacidad (personas con déficit total o parcial de la percepción que se evalúa de la pérdida del audio en cada oído), la cual forma parte de la CIF establecida por la OMS, 2001, las actividades se llevaron a cabo, bajo la anuencia de directivos, profesorado, padres y tutores del estudiantado con discapacidad auditiva, autorizando consentimiento informado por los sujetos participantes.

Los contenidos fueron sobre herramientas de trabajo en entorno virtual, la comunicación fue mediante la lengua de señas mexicana para hacer efectiva su comprensión, brindar retroalimentación, así como llevar a cabo instrucciones claras y sencillas (Serafín y González, 2011), se tomó en cuenta del contexto educativo mexicano, los lineamientos para la asistencia educativa de los estudiantes sordos que cursan Educación Básica, parten del Modelo Educativo Bilingüe-Bicultural establecido por la Secretaría de Educación Pública (SEP, 2012).

Con el fin de mejorar la motricidad a través de la clase de EF para estudiantes con discapacidad auditiva, la capacitación tomo en cuenta contenidos siguiendo recomendaciones y directrices de investigaciones realizadas en niños con discapacidad auditiva en México (Ochoa-Martínez, 2020b; Ochoa-Martínez, 2020c; Ochoa-Martínez y Alarcón, 2019; Ochoa-Martínez, Hall-López, Carmona, Morales, Alarcón, y Sáenz-Lopez, 2019; Ochoa-Martínez, Hall-López, Carmona, Reyes, Sáenz-López y Conde, 2018), enfocadas en una serie de tareas para desarrollarse a distancia, las cuales se centraron en trabajar las habilidades de coordinación, con implementos y materiales que promueven la sistematización, principalmente la motricidad fina, motricidad gruesa, equilibrio dinámico y equilibrio estático mediante actividades sincrónicas y asincrónicas utilizando los softwares Google meet ${ }^{\circledR}$ y WhatsApp ${ }^{\circledR}$ para las sesiones de EF.

Al finalizar la capacitación, de los Licenciatura en Actividad Física y Deporte, se realizó una planeación con fecha y hora para realizar las clases de EF en entorno virtual, para trabajar a distancia con los estudiantes con discapacidad auditiva durante 2 sesiones durante 6 semanas, en la que los padres de familia o tutores de los estudiantes estuvieran presentes para desarrollar las actividades durante un tiempo de 30 minutos.

\section{Análisis estadístico}

Se determinó el análisis estadístico descriptivo y el porcentaje de diferencia en los valores de la escala calculando el porcentaje de diferencia ( $\mathrm{A} \%)$ ([(Media-2Media-1)/Media-1]x 100).Y como estadística inferencial se realizó el análisis T-student para muestras relacionadas, utilizando el Programa Estadístico para las Ciencias Sociales (SPSS®), versión 25.

\section{Resultados}

La estadística descriptiva de las variables estudiadas a partir de las evaluaciones con la escala de creencias

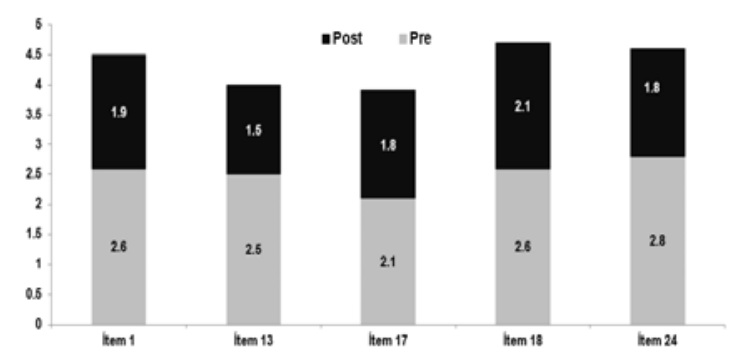

Figura 1.- Creencias de control percibido, antes y después de la intervención en los sujetos participantes. Nota: Factores de creencias de control percibido. Pre $2.5 \pm 0.2$ Post $1.8 \pm 0$. (-28 ?\%) (ítems $1,13,17,18$ y 24); 1 . No creo necesario cambiar mi forma de comportarme porque haya en clase alumnos con discapacidad en las clases de Educación Física. 13. Me sentiría incómodo trabajando con compañeros con discapacidad. 17. Las actitudes sociales hacia las personas discapacitadas pueden cambiarse en la escuela.18. No me siento preparado para tener un alumno con discapacidad en mi aula. 24. Puedo favorecer la atención a la diversidad variando la manera de hacer entender a los compañeros dentro del aula de Educación Física. 
hacia la atención a la discapacidad en actividad física (González y Baños, 2012), son presentadas en las figuras 1 a la 4, dividiendo las cuatro sub escalas con los factores de: 1.-Creencias de control percibido, 2.-Creencias sobre la actitud, 3.-Creencias generalizadas y 4.-Creencias intencionales.

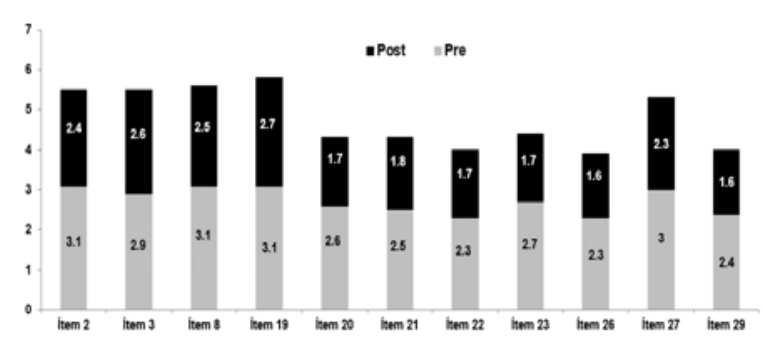

Figura 2.- Creencias sobre la actitud, antes y después de la intervención en los sujetos participantes.

Nota: Factores de creencias sobre la actitud. Pre $2.7 \pm 0.3$ Post $2.1 \pm 0.4$ (-22 ?\%) (Ítem $2,3,8,19,20,21,22,23,26,27,29$ y 30); 2 . Los alumnos con discapacidad se sienten más cómodos en las aulas de Educación Especial. 3. La atención a la discapacidad va dirigida a los alumnos que presentan alguna discapacidad. 8. Sobre discapacidad preferiría que me asesorara un especialista a un compañero. 19. En una clase ordinaria no debería haber más de un alumno con discapacidad. 20. En una clase de actividad física heterogénea el maestro no puede dar una respuesta educativa a cada alumno. 21. No me importaría trabajar junto a personas con discapacidad. 22. La inclusión

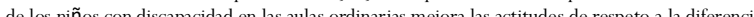
de los niños cresterencia en sus compañeros. 23. Los alumnos con discapacida pueden colaborar con compañeros en las actividades de clase. 26. Trabajar en equipo entre compañeros, en las escuelas, hoy en día es imposible. 27. Los alumnos con discapacidad necesitan un currículum diferente a los demás alumnos. 29. La inclusión de los niños con discapacidad en las aulas ordinarias mejora las actitudes de respeto a la diferencia en sus compañeros. 30. Los alumnos con discapacidad pueden colaborar con sus compañeros en las actividades de la clase.

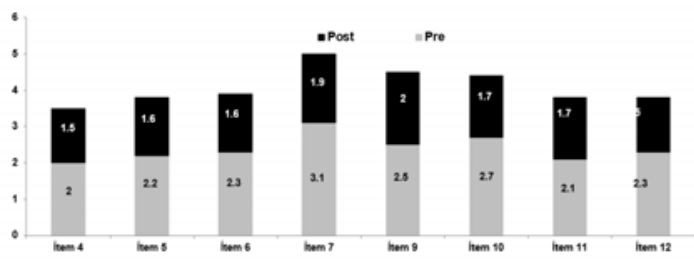

Figura 3.-Creencias generalizadas, antes y después de la intervención en los sujetos participantes. Nota: Factores de creencias generalizadas. Pre $2.4 \pm 0.3$ Post $1.7 \pm 0.1$ (-29 ?\%) (Ítems $4,5,6,7,9,10,11$ y 12); 4 . Sería conveniente que los alumnos con discapacidad compartieran aula sólo con compañeros que tengan las mismas características. 5. Los centros espećficos son escuelas mejor equipadas y con mejores profesionales para atender la discapacidad de los alumnos. 6. No se puede ser tan exigente en las clases de EF con los alumnos con discapacidad alumnos. 6. No se puede ser tan exigente en las clases de EF con los alumnos con discapacidad
que con los demás. 7. El profesor de apoyo debe encargarse del aprendizaje de los niños con discapacidad y el profesor tutor del resto de los alumnos. 9. Los alumnos con alguna discapacidad deben asistir a centros especiales. 10. Los mayores problemas que tendría en clase con niños con discapacidad serían de entendimiento. 11. La integración favorece a los alumnos con discapacidad, pero perjudica a los demás. 12. La presencia del profesor de apoyo dentro de mi aula entorpecería mi trabajo con los demás alumnos.

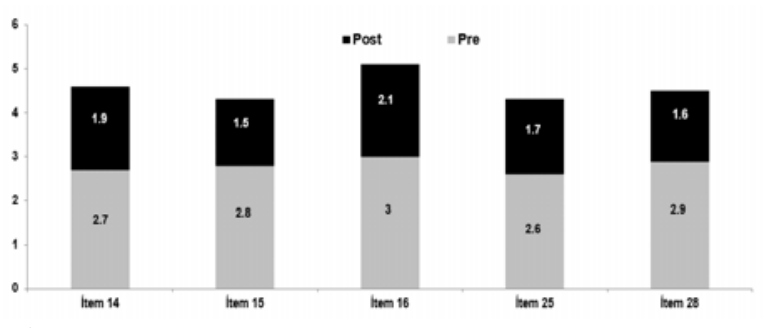

Figura 4.- Creencias intencionales, antes y después de la intervención en los sujetos participantes.

Nota: Factores de creencias intencionales. Pre $2.8 \pm 0.1$ Post $1.8 \pm 0.2(-36 \Delta \%)$ (Ítems $14,15,16,25$ y 28); 14. Prefiero trabajar en aulas con alumnado homogéneo. 15. Preferiría no tener alumnos con discapacidad en mi clase. 16. Estaría dispuesto a emprender una iniciativa tener alumnos con discapacidad en mi clase. 16. Estaria dispuesto a emprender una iniciativa
en mi centro para que alumnos y profesores aprendieran en sistema Braille o alguna adaptación para la EF. 25. Debería apoyar más en actividades en las que puedan participar todos los alumnos, incluidos los que presentan discapacidad. 28. El tipo de enseñanza que necesita un alumno con discapacidad lo determina el nivel de discapacidad y de apoyo que pueda tener en el aula por parte de compañeros y/ó profesores.

\section{Discusión}

Al evaluar las creencias hacia la atención a la discapacidad en actividad física de los alumnos de la Li- cenciatura en Actividad Física y Deportes que realizaron práctica docente de Educación Física en la modalidad a distancia, dirigida a escolares con discapacidad auditiva, en promedio los 30 ítems del instrumento fue $2.6 \pm 0.2$ y después de la intervención $1.9 \pm 0.2$ con un porcentaje de cambio de -29 Ä \%, el análisis estadístico inferencial utilizando el test t-Student para muestras independientes reportó diferencias significativas ( $\mathrm{p}<0.05)$. González y Baños (2012) evaluaron las creencias hacia la atención a la discapacidad en actividad física, en estudiantes de secundaria que practicaron deportes con personas con discapacidad visual, al compararlo con la presente investigación nuestros resultados, fueron más cercanos a 1 de la escala reflejando una actitud positiva ante las personas con discapacidad. Al revisar el estado de la cuestión, una investigación realizada en el ámbito de la actividad física en EF reportó en el profesorado actitudes moderadamente positivas hacia la inclusión de alumnos con discapacidad (Solís y Borja, 2020), de igual forma el resultado de nuestra investigación es congruente con el estudio realizado por OchoaMartínez, 2020a), que evaluó la actitud en futuros Licenciados en Actividad Física y Deporte que participaron en programa de prescripción de ejercicio físico a jóvenes universitarios con discapacidad auditiva, los resultados mostraron también mejora de la actitud, sin embargo se utilizó otro instrumento de evaluación para valorar la actitud, pero a pesar de la diferencia metodológica de los instrumentos tanto en actividades sincrónicas y asincrónicas las actividades formativas prácticas que involucran contacto con personas con discapacidad mejoran la actitud en el estudiantado.

El estudio presenta limitaciones al no notar con un grupo control, adicionalmente los resultados obtenidos aplican a un contexto educativo especifico, pero al contar con pocas referencias en el estado del arte de estudios realizados durante la pandemia por COVID-19, la presente investigación sirve de experiencia formativa, al profesorado de EF que trabaja en torno a la discapacidad auditiva en futuros abordajes pedagógicos, tomando en cuenta, que para los estudiantes con discapacidad auditiva que presentan un daño en el sistema vestibular diagnosticado, ya sea por una enfermedad o un padecimiento congénito, lo cual representa dificultades para aprender su propia lengua, seguir con el aprovechamiento de las enseñanzas educativas (Franco y Panhoca, 2008; Gómez, 2014), adecuándose a las necesidades actuales, trabajando a distancia y realizando adecuaciones curriculares debido a la crisis de salud por COVID-19 (Hall y Ochoa-Martínez, 2020; Hall- López, 
2020; Hall-López, et al. 2021).

Por otra parte, como línea de investigación en el futuro, el estudio de factores socio afectivos en la educación física alternos a la actitud como lo es la sensibilización (Abellán, Segovia, Gutiérrez y García, 2022) en el estudiantado con discapacidad, podrían mejorar el proceso enseñanza aprendizaje.

\section{Conclusión}

A partir de lo planteado anteriormente, se destaca que las experiencias formativas en licenciatura mejoran las creencias hacia la atención a la discapacidad en actividad física, trabajando en entornos de enseñanza aprendizaje a distancia sincrónico y asincrónico, por parte del Licenciado en Actividad Física y Deporte, lo cual es positivo para el futuro profesional, que pueda contar con adecuadas competencias apoyado de las tecnologías de la información y comunicación para el desarrollo de intervenciones efectivas en poblaciones con diferentes tipos de discapacidad en EF en el sector público y privado, tomando en cuenta la posibilidad de futuros rebrotes de infección por COVID-19, donde se requiera en mayor medida estos procedimientos de aprendizaje.

\section{Referencias}

Abellán, J., Segovia,Y., Gutiérrez, D., \& García López, L. M. (2022). Sensibilización hacia la discapacidad a través de un programa integrado de Educación Deportiva y Aprendizaje-Servicio (Disability awareness through an integrated program of sport education and servicelearning). Retos, 43, 477-487. doi.org/10.47197/ retos.v43i0.86625

Alcaín, E., y Medina-García, M. (2017). Hacia una educación universitaria inclusiva: realidad y retos. Revista Digital de Investigación en Docencia Universitaria, 11(1), 419. doi.org/10.19083/ridu. 11.530

Baena-Morales, S., López-Morales, J., y García-Taibo, O. (2020). La intervención docente en educación física durante el periodo de cuarentena por COVID-19, Retos, 39, 388-395. doi.org/10.47197/retos.v0i39.80089

Barboza, C., Ramos, A., Abreu, P., y Castro H. (2019). Physical Education: Adaptations and Benefits for Deaf Students. Creative Education, 10, 714-725. doi.org/10.4236/ ce.2019.104053

Blocken, B., Malizia, F., vanDruenen.,T., y Marchal,T. (2020). Towards aerodynamically equivalent COVID-19 $1.5 \mathrm{~m}$ social distancing for walking and running. Urban Physics, Wind Engineering \& SportsAerodynamics. Preprint. https: // www.euroga.org/system/1/user_files/files/000/
$045 / 111 / 45111 / 150 \mathrm{~d} 3060 \mathrm{c} /$ original / Social_Distancing_v20_White_Paper.pdf

Camaño, L., Rodríguez,Y., Rojas, T. M., Erazo, G. F., y Pancho, T. L. (2019). Estudio de las actitudes de docentes hacia la discapacidad en una universidad ecuatoriana. Revista Espacios, 40 (39), 17. https: / /www.revistaespacios.com/ a19v40n39/a19v40n39p17.pdf

Chen, P., Mao, L., Nassis, G. P., Harmer, P., Ainsworth, B. E., y Li, F. (2020). Coronavirus disease (COVID-19): The need to maintain regular physical activity while taking precautions. Journal of sport and health science, 9(2), 103104. doi.org/10.1016/j.jshs.2020.02.001

Franco, E. S., y Panhoca, I. (2008). Vestibular function in children underperforming at school. Brazilian Journal of Otorhinolaryngology, 74(6), 815-25. https:// www.sciencedirect.com/science/article/pii/ S1808869415301415

Fiorini, M. L.S., y Manzini, E. J. (2018). Strategies of Physical Education Teachers to Promote the Participation of Students with Hearing Impairment in Classrooms. Revista Brasileira de Educação Especial, 24(2), 183-198. doi.org/ 10.1590/s1413-65382418000200003

González, J y Baños, L. M. (2012). Estudio sobre el cambio de actitudes hacia la discapacidad en clases de actividad física. Cuadernos de Psicología del Deporte, 12(2), 101-108. https: / / revistas.um.es/cpd/article/view/170371

González, R., Gastélum-Cuadras, G., Velducea,W., González, J. B., y Domínguez, S. (2021). Análisis de la experiencia docente en clases de Educación Física durante el confinamiento por COVID-19 en México. Retos, 42, 1-11. doi.org/10.47197/retos.v42i0.86242

Gómez, R. M. (2014). La inclusión de la persona sorda a la Educación Superior. Revista Latinoamericana de Educación Inclusiva, 8(1), 93-108. http://www.rinace.net/rlei/ numeros/vol8-num1/art6.pdf

Hall-López, J.A., Ochoa-Martínez, P.Y., y Alarcón, E. I. (2021). Alternativas durante el covid-19, para profesorado universitario dedicado a la enseñanza de la educación física. Cuerpo, CulturaY Movimiento, 11(1), 4-13. doi.org/ 10.15332/2422474X.6464

Hall-Lopez, J. A. (2020). Physical activity levels in physical education teachers before and during school suspension brought by the covid-19 quarantine. Facta Universitatis, Series Physical Education and Sport, 19(2), 179-188. doi.org/ 10.22190/FUPES200607045H

Hall-López, J., y Ochoa-Martínez, P. (2020). Enseñanza virtual en educación física en primaria en México y la pandemia por COVID-19. Ciencias De LaActividad Física UCM, 21(2), 1-7. doi.org/10.29035/rcaf.21.2.4

Hortigüela-Alcalá, D., Garijo,A., y Pérez-Pueyo, Á. (2021). La Educación Física en el contexto COVID-19. Un relato de profesores de diferentes etapas educativas. Retos, 41, 
764-774. doi.org/10.47197/retos.v41i0.86368

Jung, J., Leung, W., Schram, B. M., yYun, J. (2018). MetaAnalysis of Physical Activity Levels in Youth With and Without Disabilities. Adapted physical activity quarterly: APAQ, 35(4), 381-402. doi.org/10.1123/apaq.20170123

Lebrasseur,A., Fortin-Bédard, N., Lettre, J., Bussières, E. L., Best, K., Boucher, N., Hotton, M., Beaulieu-Bonneau, S., Mercier, C., Lamontagne, M. E., y Routhier, F. (2021). Impact of COVID-19 on people with physical disabilities: A rapid review. Disability and health journal, 14(1), 101014. doi.org/10.1016/j.dhjo.2020.101014

Li, R., Sit, C.H.P., Yu, J.J., Duan, J., Fan, J., Mckenzie,T., y Wong, S. (2016). Correlates of physical activity in children and adolescents with physical disabilities: A systematic review. Preventive Medicine, 89, 184-193. doi.org/ 10.1016/j.ypmed.2016.05.029.

Lippi, G., Henry, B. M., y Sanchis-Gomar, F. (2020). Physical inactivity and cardiovascular disease at the time of coronavirus disease 2019 (COVID-19). European Journal of Preventive Cardiology, O(0), 1-3. doi.org/10.1177/ 2047487320916823

Ochoa-Martínez, P. (2020a). Experiencia didáctica en educación física para la mejora de actitudes hacia la discapacidad auditiva en futuros profesionales de la actividad física y deporte, Retos, 40, 174-179. doi.org/10.47197/ retos.v1i40.81296

Ochoa-Martínez, P.Y.(2020b). Pedagogical Strategies for Gross and Fine Motor Skills Learning Through Physical Education: Intervention in Students with Hearing Impairment. Revista Brasileira de Educação Especial, 26(4), 567-570. doi.org/10.1590/1980-54702020v26e0063

Ochoa-Martínez, P.Y. (2020c). Physical education in school children with partial or complete hearing loss to improve gross motor skill development. Revista Espacios, 41(46) 1-10. doi.org/10.48082/espacios-a20v41n46p01

Ochoa-Martínez, P.Y., y Alarcón, E. I. (2019). Edad motora en escolares con discapacidad auditiva como diagnóstico de intervención educativa en educación física., En F. Santillán (coord.), El pensamiento del profesor investigador vínculo entre la teoría y la práctica, (pp. 171-179). Guadalajara (México): Centro de Estudios e Investigaciones para el Desarrollo Docente CENID. http: / / www.cenid.org.mx/libros/libros19/libro014/\#p=1

Ochoa-Martínez, P.Y., Hall-López, J.A., Carmona,A., Morales, M.,Alarcón, E., y Sáenz-Lopez, P. (2019). Effect of an Adapted Program of Physical Education in Children with Hearing Disability on Motor Coordination. MHSalud: Revista En Ciencias Del Movimiento HumanoY Salud, 16(2), 111. doi.org/10.15359/mhs. 16-2.2

Ochoa-Martínez, P.Y., Hall-López, J.A., Carmona López,A., Reyes Castro, Z., Sáenz-López Buñuel, P., y Conde, C.
(2018). Análisis comparativo de un programa educación física en niños con discapacidad auditiva sobre la edad motora equivalente Retos, 35, 310-313. https:// recyt.fecyt.es/index.php/retos/article/view/67190

Organización de las Naciones Unidas para la Educación, la Ciencia y la Cultura (Unesco) (2015). Educación física de calidad. Guía para los responsables políticos. Recuperado de http: / / unesdoc.unesco.org/images/0023/002313/ 231340S.pdf.

Organización Mundial de la Salud(OMS). Clasificación internacional del funcionamiento, de la discapacidad y de la salud (International Classification of Functioning, Disability and Health). 2001. website: https: //www.who.int/ classifications/icf/en/

Rundle,A. G., Park,Y., Herbstman, J., Kinsey, E.W., yWang,Y. C. (2020) COVID-19-Related School Closings and Risk of Weight Gain Among Children. Obesity (Silver Spring), 28(6), 1008-1009. doi.org/10.1002/oby. 22813

Secretaría de Educación Pública SEP Orientaciones para la atención educativa de alumnos sordos que cursan la Educación Básica, desde el Modelo Educativo BilingüeBicultural (1ra. Ed.). México, 2012. https:// www. educacionespecial.sep.gob.mx/2016/ index_disca.html

Serafín, M. E., y González, R. (2011). Manos con voz diccionario de lengua de señas mexicana. Una herramienta indispensable para conocer el lenguaje de señas (1 ra. Ed.). Consejo Nacional para Prevenir la Discriminación (CONAPRED) México. 2011. https:// w w w. C o n a p r e d. o r g. m x / index.php?contenido $=$ documento\&id $=2618 i d$ _opcion $=\& o p=215$ Solís, P., y Borja,V. (2020). Actitudes del profesorado de Educación Física hacia la inclusión de alumnos con discapacidad. Retos, 39, 7-12. https:/ / recyt.fecyt.es/ index.php/retos/article/view/77841

Varguillas, C.S., y Bravo, P. C. (2018).Atención al estudiante con discapacidad. Un análisis descriptivo. Caso Universidad Pedagógica Experimental Libertador (UPEL) Venezuela. Revista Espacios, 39(49), 19. http:// www.revistaespacios.com/a $18 v 39$ n $49 /$ a18v39n49p19.pdf

Webster, C.A., Webster, L., Russ, L., Molina, S., Lee, H., y Cribbs, J. (2015). A systematic review of public healthaligned recommendations for preparing physical education teacher candidates. Research quarterly for exercise and sport, 86(1), 30-39. doi.org/10.1080/ 02701367.2014 .980939 